Pacific Journal of Mathematics

EIGENFUNCTION EXPANSIONS FOR SELFADJOINT
INTEGRO-DIFFERENTIAL OPERATORS 


\title{
EIGENFUNCTION EXPANSIONS FOR SELF-ADJOINT INTEGRO-DIFFERENTIAL OPERATORS
}

\section{ROBERT CARLSON}

\begin{abstract}
Let $\mathscr{L}$ be a self-adjoint ordinary differential operator on a Hilbert space $L^{2}(\mathscr{F}), \mathscr{I}$ an open interval, while $\mathscr{K}$ will denote a bounded self-adjoint operator on the same space. An eigenfunction expansion associated with $H=\mathscr{L}+\mathscr{K}$ is developed when $\mathscr{K}$ is an integral operator whose kernel $K(x, y)$ has compact support in $\mathscr{F} \times \mathscr{F}$. It is assumed that if $\mathscr{L} f=\sum_{k=0}^{n} a_{k}(x) D^{k} f$, where $D=(d / d x)$, then $a_{k} \in C^{k}(\mathscr{I})$, $k=0, \cdots, n$, and $a_{n}(x) \neq 0$ for $x \in \mathscr{I}$.
\end{abstract}

In the previously studied cases, when $\mathscr{K}=0$, one can choose a specific basis for the space of solutions to the equation

$$
\sum_{k=0}^{n} a_{k}(x) D^{k} s(x, l)=l s(x, l),
$$

and, by analysis of the resolvent of the operator $\mathscr{L}$, prove that the mapping $f \rightarrow \hat{f}$ defined by $\hat{f}(\nu)=\int_{\mathscr{S}} f(x) s(x, \nu) d x$ is an isometric mapping from $L^{2}(\mathscr{I})$ onto $L^{2}(d \rho)$, where $d \rho$ is a matrix-valued measure on the line. Here $s(x, \nu)$ is a vector-valued function whose components are the elements of the distinguished basis. With major modifications this plan is carried out to give similar results for the operators $\mathscr{L}+\mathscr{K}$.

The analysis begins with a study of self-adjoint operators formally agreeing with $\mathscr{L}$ on $L^{2}(\mathscr{J}), \mathscr{J}$ a compact subinterval of $\mathscr{F}$, and their perturbations by operators $\mathscr{K}$ as above. With mild restrictions on the domains, these self-adjoint operators have compact resolvents. This allows us to exhibit analytic bases for the solutions of the integro-differential equations $L f+\int_{\sigma} K(x, y) f(y) d y=l f$. These bases provide an analog of the usual basis of solutions to the differential equation $L f_{i}=l f_{i}$ which satisfies $f_{i}^{(j-1)}(c, l)=\delta_{i j}$ for some $c \in \mathscr{F}$. The resolvents of operators $\mathscr{H}$ as above share most of the smoothness properties of the resolvent of the differential operator $\mathscr{L}$. In particular if $\mathscr{L}$ has order at least two, the resolvent of $H$ will be an integral operator, $R_{H}(l) f=\int_{l} R_{H}(x, y, l) f(y) d y$, whose kernel is continuous in the pair $(x, y)$ and analytic in $l$.

These results allow us to use the methods of Coddington [2], [4], and Coddington and Dijksma [5] to derive eigenfunction expansions closely paralleling those known for ordinary differential operators. Formally the difference arises when eigenspaces of $H$ have dimension 
greater than the order of $\mathscr{L}$. The corresponding set of eigenvalues is shown to be a closed countable set of real numbers, and the eigenspaces have finite dimension. This contrasts strongly with the pathology possible if no restrictions are made on the support of the kernel of the perturbation $\mathscr{K}$. A discussion of this problem is in $\S$ VII.

Fixing notation, denote the real and complex numbers by $R$ and $C$ respectively, and let $C_{0}$ be the set of complex numbers with nonzero imaginary part. If $H$ is a Hilbert space operator its null space, range and domain are $\nu(H), R(H)$, and $D(H)$ respectively. The operator $(H-l I)^{-1}$, defined in the resolvent set $\rho(H)$, will be denoted by $R_{H}(l)$, and $\sigma(H)$ will be the spectrum of $H . H^{*}$ will be the adjoint of $H$. The formal operator $\sum_{k=0}^{n} a_{k} D^{k}$ is denoted by $L$.

This work was part of the author's dissertation, written under Earl Coddington. I would like to take this opportunity to thank him for his assistance.

II. Abstract preliminaries. If $B$ and $T$ are Hilbert space operators satisfying $D(T) \subset D(B)$, and if there are constants $\alpha, \beta$ such that $\|B f\| \leqq \alpha\|f\|+\beta\|T f\|$ for all $f \in D(T)$, then we say that $B$ is $T$ bounded. If we can choose $\beta<1$ we say $B$ has $T$-bound less than one. The following theorem, due to Rellich (Kato [9]), shows that $\mathscr{L}+\mathscr{K}$ is self-adjoint.

THEOREM 2.1. If $B$ is symmetric and T-bounded with T-bound less than one, and if $T$ is self-adjoint, then $T+B$ is self-adjoint.

The next well known result can be found in [8] (p. 121).

Lemma 2.ii. Let $A$ and $B$ be closed operators on a Hilbert space. If $D(B) \subset D(A)$, then there is a constant $K$ such that $\|A x\| \leqq$ $K(\|x\|+\|B x\|)$ for all $x \in D(B)$.

In what follows we will need to know that certain symmetric operators have self-adjoint extensions with compact resolvents. For this reason we introduce the notion of compact embedding. We say that a Hilbert space $\mathscr{H}$ is compactly embedded in $L^{2}(\mathscr{F})$ if each element of $\mathscr{H}$ is in $L^{2}(\mathscr{F})$ and if every sequence $\left\{f_{n}\right\} \subset \mathscr{H}$ that is bounded in the norm of $\mathscr{H}$ has a convergent subsequence in $L^{2}(\mathscr{F})$.

THEOREM 2.iii. Let $H$ be a self-adjoint operator on $L^{2}(\mathscr{F})$. The following are equivalent:

1. $D(H)$ in the graph topology is compactly embedded in $L^{2}(\mathscr{F})$,

2. the spectrum of $H$ consists of isolated eigenvalues of finite 
multiplicity,

3. $(H-\mathfrak{l} I)^{-1}$ is compact for $\mathfrak{l} \in \rho(H)$.

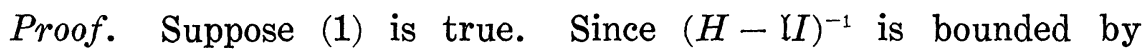
$1 / d(\mathfrak{l}, \sigma(H))$, we have

$$
\left\|H(H-\mathfrak{l} I)^{-}\right\| f+\left\|(H-\mathfrak{\imath} I)^{-1} f\right\| \leqq\left[1+\frac{\mathfrak{l}+1}{d(\mathfrak{l}, \sigma(H))}\right]\|f\|,
$$

or $(H-\mathfrak{l} I)^{-1}$ is bounded from $L^{2}(\mathscr{J})$ to $D(H)$. This proves (3). Moreover, that (3) implies (2) is well known.

To see that (2) implies (1), let $\lambda$ be real and in the resolvent set of $H$. It is easily verified that $\mu$ is an eigenvalue of $H$ of multiplicity $m$ if and only if $1 /(\mu-\lambda)$ is an eigenvalue of $(H-\lambda I)^{-1}$ of multiplicity $m$. Thus $(H-\lambda I)^{-1}$ has a bounded set of eigenvalues of finite multiplicity clustering only at zero. By the spectral theorem for self-adjoint operators, $(H-\lambda I)^{-1}$ is compact. In fact, to approximate $(H-\lambda I)^{-1}$ uniformly within $\varepsilon$ by a finite rank operator simply compose $(H-\lambda I)^{-1}$ with the orthogonal projection onto the eigenspaces corresponding to the eigenvalues of norm greater than or equal to $\varepsilon$.

Thus, since $H-\lambda I$ is bounded below, the set of $f \in D(H)$ such that $\|f\|+\|H f\| \leqq 1$ is contained in the set of $f$ such that $\|(H-\lambda I) f\| \leqq K$, for some constant $K$. Now this set of $f \in D(H)$ is just $(H-\lambda I)^{-1}$ of the $K$-ball, a set with compact closure.

Recall that $\mathscr{L}$ is a self-adjoint ordinary differential operator on $L^{2}(\mathscr{I})$ whose domain is assumed to include $C_{0}^{\infty}(\mathscr{J})$, the infinitely differentiable functions with support in $\mathscr{F}$. Let $H$ be another selfadjoint operator satisfying $D(H)=D(\mathscr{L})$. The closure of the restriction of $H$ (resp. $\mathscr{L}$ ) to $C_{0}^{\infty}(\mathscr{F})$ will be denoted by $H_{0}\left(\right.$ resp. $\left.\mathscr{L}_{0}\right)$ and called the minimal operator associated with $H($ resp. $\mathscr{L})$. Since, by (2.ii), the graph topologies of $D(H)$ and $D(\mathscr{L})$ coincide, $D\left(H_{0}\right)=D\left(\mathscr{L}_{0}\right)$. Considering these operators as subspaces of the Hilbert space $L^{2}(\mathscr{J}) \oplus$ $L^{2}(\mathscr{S})$ we have:

LEMMA 2.iv. The dimension of $H_{0}^{*} \theta H_{0}$ is twice the dimension of $\mathscr{L} \theta \mathscr{L}_{0}$.

Proof. It suffices to show that the dimensions of $H \theta H_{0}$ and $\mathscr{L} \theta \mathscr{L}_{0}$ are equal. Define a linear mapping $Q$ from $\mathscr{L} \theta \mathscr{L} \mathscr{L}_{0}$ to $H \theta H_{0}$ as follows. If $\{f, \mathscr{L} f\} \in \mathscr{L} \theta \mathscr{L}_{0}$ define $Q\{f, \mathscr{L} f\}$ to be the orthogonal projection of $\{f, H f\}$ onto $H \theta H_{0}$. Since $\{f, \mathscr{L} f\} \in \mathscr{L} \theta \mathscr{L}_{0}, f \in D\left(\mathscr{L}_{0}\right)$ if and only if $f=0$, hence $Q$ is an injection from $\mathscr{L} \theta \mathscr{L}_{0}$ into $H \theta H_{0}$. This implies 
that the dimension of $H \theta H_{0}$ is no smaller than that $\mathscr{L} \theta \mathscr{L}_{0}$. Reversing the argument yields the result.

The next pair of lemmas generalizes work in Dunford and Schwartz ([7], p. 1400). The notation is as in [3]. For closed operators we are simply identifying the operator with its graph. Let $\mathscr{C}$ be a Hilbert space.

Lemma 2.v. Let $S$ be a symmetric subspace in $\mathscr{H} \oplus \mathscr{H}$ with adjoint subspace $S^{*}$. If $N=\left\{\{f, 0\} \in S^{*}\right\}$, then $S+N=\{\{f, g\}+$ $\{h, k\} \mid\{f, g\} \in S,\{h, k\} \in N\}$ is symmetric.

Proof. As subspaces $(S+N)^{*}=S^{*} \cap N^{*}$. Since $N=\nu\left(S^{*}\right) \oplus\{0\}$, $N^{*}=\mathscr{H} \oplus\left(\nu\left(S^{*}\right)\right)^{\perp}=\mathscr{H} \oplus(R(S))^{c}$. Thus $(S+N)^{*}=S^{*} \cap(\mathscr{H} \oplus$ $\left.(R(S))^{c}\right)$. Obviously both $S$ and $N$ are subspaces of $(S+N)^{*}$, hence $(S+N) \subset(S+N)^{*}$.

Define $M^{ \pm}=\left\{\{f, \pm i f\} \in S^{*}\right\}$. Then it is known ([3]) that every self-adjoint extension $H$ of $S$ is of the form $H=S \oplus(I-V) M^{+}$ where $I$ denotes the identity on $\mathscr{H} \oplus \mathscr{H}$ and $V$ is an isometry from $M^{+}$onto $M^{-}$.

Lemma 2.vi. Suppose $N \cap S=\{0\}$ and dimension $M^{+}=$dimension $M^{-}<\infty$. Then there is a self-adjoint subspace extension $H$ of $S$ such that $N \cap H=\{0\}$.

Proof. Let $H_{1}$ be any self-adjoint extension of $S+N$. We have the usual decomposition $H_{1}=S \oplus\left(I-V_{1}\right) M^{+}$, and since $S+N$ is symmetric there is a subspace $W \subset M^{+}$such that $S+N=S \oplus$ $\left(I-U_{1}\right) W$, where $U_{1}$ is an isometry of $W$ into $M^{-}$(see Coddington [3]). First we show that $U_{1}=V_{1} \mid W$. Since $S+N \subset H_{1}$ every element in $(S+N) \theta S$ can be written as $\left(I-V_{1}\right) w_{1}=\left(I-U_{1}\right) w_{2}$. The orthogonality of $M^{+}$and $M^{-}$implies $w_{1}=w_{2}$, and hence $V_{1} w_{1}=U_{1} w_{1}$.

Thus $S+N=S \oplus\left(I-V_{1}\right) W$. Define an isometry $V$ from $M^{+}$to $M^{-}$by requiring $V=V_{1}$ on $W^{\perp}$, where $W^{\perp}=M^{+} \theta W$, and $V=-V_{1}$ on $W$. Extend $V$ linearly to get an isometry. Define $H=S \oplus$ $(I-V) M^{+}$, and observe that $(I-V) M^{+}=(I-V) W \oplus(I-V) W^{\perp}$.

To establish $N \cap H=\{0\}$ we let $\{f, 0\} \in S \oplus(I-V) M^{+}$. Since $(I-V) W^{\perp}=\left(I-V_{1}\right) W^{\perp}$, and $\left(I-V_{1}\right) W^{\perp}$ is orthogonal to $S+N$, we must have $\{f, 0\} \in S \oplus(I-V) W$. Consequently there are elements $s_{1} \in S, h \in W$ and $k \in V W$ such that $\{f, 0\}=s_{1}+h-k$. Since the isometry $V$ is $-V_{1}$ on $W$, the element $s_{1}+h+k \in S \oplus\left(I-V_{1}\right) W=$ $S+N$. Thus there is an $s_{2} \in S$ such that $\left(s_{1}-s_{2}\right)+h+k \in N$. Since $N$ is a subspace and $\{f, 0\} \in N,\left(2 s_{1}-s_{2}\right)+2 h \in N$. Since $h$ 
is now in $(S+N) \cap M^{+}$, it follows that $h=0$ since $S+N$ cannot have $+i$ as an eigenvalue. This further implies $k=0$, so that $f$, now in $S \cap N$, must be 0 .

III. Solutions of $L f+\int_{\mathscr{S}} K(x, y) f(y) d y=\mathfrak{l} f$. If $S$ is a symmetric ordinary differential operator agreeing with $L=\sum_{k=0}^{n} a_{k} D^{k}$ on its domain, then the finite dimensional space $\nu\left(S^{*}-\mathfrak{I} I\right), \mathfrak{l} \in C$, is contained in the finite dimensional space of solutions of the associated differential equation $(L-\mathfrak{l}) s(x, \mathfrak{l})=0 . \quad$ A basic existence theorem for ordinary differential equations guarantees bases for the spaces of solutions of $(L-\mathfrak{l}) s(x, \mathfrak{l})=0$ which are, for fixed $x$, entire functions of $\mathfrak{l}$. Here we develop analogous results for certain symmetric operators with finite deficiency indices.

TheOREM 3.i. Let $S$ be a densely defined symmetric operator in $a$ Hilbert space $\mathscr{K}, H$ a self-adjoint extension of $S$ in the same Hilbert space, and dimension $\nu\left(S^{*}-\mathfrak{l} I\right)<\infty$ for $\mathfrak{l} \in \rho(H)$. Then there is a basis for $\nu\left(S^{*}-\mathfrak{l} I\right)$ which is analytic on path-connected components of $\rho(H)$.

Proof. Fix $\mathfrak{l}_{0} \in \rho(H)$ and let $\theta_{i}\left(\mathfrak{l}_{0}\right), i=1, \cdots, n$ a basis for $\boldsymbol{\nu}\left(S^{*}-\mathfrak{l}_{0} I\right)$. For $\mathfrak{l}$ in the same path component as $\mathfrak{I}_{0}$ define functions

$$
\theta_{i}(\mathfrak{l})=\left[I+\left(\mathfrak{l}-\mathfrak{l}_{0}\right) R_{H}(\mathfrak{l})\right] \theta_{i}\left(\mathfrak{l}_{0}\right) \text { for } \quad i=1, \cdots, n .
$$

One can verify directly that $\theta_{i}(\mathfrak{l}) \in \mathcal{\nu}\left(S^{*}-\mathfrak{l} I\right)$, and, as long as $\left\|\left(\mathfrak{l}-\mathfrak{l}_{0}\right) R_{H}(\mathfrak{l})\right\|<1,\left\{\theta_{i}(\mathfrak{l})\right\}_{i=1}^{n}$ will give a basis for $\nu\left(S^{*}-\mathfrak{l} I\right)$. What we intend to show is that $\left\{\theta_{i}(\mathfrak{l})\right\}_{i=1}^{n}$ is in fact a basis for $\nu\left(S^{*}-\mathfrak{l} I\right)$ for all $\mathfrak{l}$ in the same path component of $\rho(H)$ as $\mathfrak{I}_{0}$.

Pick $\mathfrak{l}_{1} \in \rho(H)$ and for each $i=1,2, \cdots, n$ define

$$
\tilde{\theta}_{i}(\mathfrak{l})=\left[I+\left(\mathfrak{l}-\mathfrak{l}_{1}\right) R_{H}(\mathfrak{l})\right] \theta_{i}\left(\mathfrak{l}_{1}\right) .
$$

Since $R_{H}(\mathfrak{l})-R_{H}(m)=(\mathfrak{l}-m) R_{H}(\mathfrak{l}) R_{H}(m)$, we have

$$
\begin{aligned}
\tilde{\theta}_{i}(\mathfrak{l})= & {\left[I+\left(\mathfrak{l}-\mathfrak{l}_{1}\right) R_{H}(\mathfrak{l})\right]\left[I+\left(\mathfrak{l}_{1}-\mathfrak{l}_{0}\right) R_{H}\left(\mathfrak{l}_{1}\right)\right] \theta_{i}\left(\mathfrak{l}_{0}\right) } \\
= & {\left[I+\left(\mathfrak{l}-\mathfrak{l}_{1}\right) R_{H}(\mathfrak{l})+\left(\mathfrak{l}_{1}-\mathfrak{l}_{0}\right) R_{H}\left(\mathfrak{l}_{1}\right)\right.} \\
& \left.+\left(\mathfrak{l}_{1}-\mathfrak{l}_{0}\right)\left(R_{H}(\mathfrak{l})-R_{H}\left(\mathfrak{l}_{1}\right)\right)\right] \theta_{i}\left(\mathfrak{l}_{0}\right) \\
= & {\left[I+\left(\mathfrak{I}-\mathfrak{l}_{0}\right) R_{H}(\mathfrak{l})\right] \theta_{i}\left(\mathfrak{l}_{0}\right)=\theta_{i}(\mathfrak{l}) . }
\end{aligned}
$$

In other words the "different" definitions of $\theta_{i}(\mathfrak{l})$ and $\tilde{\theta}_{i}(\mathfrak{l})$ agree.

Given $\mathfrak{I}_{0}$ and $\mathfrak{l}$ in the same path component of $\rho(H)$, it is possible to find a path in $\rho(H)$ from $\mathfrak{I}_{0}$ to $\mathfrak{l}$. Using the compactness of this path we can find a finite set of points $\left\{z_{i}\right\}_{i=1}^{k}$ such that $z_{i} \in \rho(H), l_{0}=z_{1}$, $l=z_{k}$ and 


$$
\left\|\left(z_{i+1}-z_{i}\right) R_{H}\left(z_{i+1}\right)\right\|<1 .
$$

Thus $\left\{\theta_{i}\left(z_{2}\right)\right\}_{i=1}^{n}$ is a basis for $\nu\left(S^{*}-z_{2} I\right)$. Now $\theta_{i}\left(z_{3}\right)$ is both $[I+$ $\left.\left(z_{3}-z_{1}\right) R_{H}\left(z_{3}\right)\right] \theta_{i}\left(z_{1}\right)$ and $\left[I+\left(z_{3}-z_{2}\right) R_{H}\left(z_{3}\right)\right] \theta_{i}\left(z_{2}\right)$. By $(\dagger)\left\{\theta_{i}\left(z_{3}\right)\right\}_{i=1}^{n}$ is a basis for $\nu\left(S^{*}-z_{3} I\right)$. We continue the induction to complete the proof.

Corollary 3.ii. Let dimension $\nu(S-\lambda I)=0$ for $\lambda \in C$, and suppose dimension $M^{+}=$dimension $M^{-}<\infty$. If $S$ has a self-adjoint extension with compact resolvent, then there is a neighborhood $U$ of $\lambda$ and a basis for $\nu\left(S^{*}-\lambda I\right)$ which is analytic in $U$.

Proof. By (2.vi) there is a self-adjoint extension $H$ of $S$ with $\lambda \in \rho(H)$. Now apply (3.i) to get the result.

Lemma 3.iii. Let $\mathscr{L}$ be a self-adjoint ordinary differential operator on $L^{2}(\mathscr{J})$, and let $\mathscr{K}$ be symmetric with $D(\mathscr{L}) \subset D(\mathscr{K})$. Assume that $\mathscr{L}+\mathscr{K}$ is self-adjoint and, if $\mathscr{L}_{0}$ is the minimal operator corresponding to $\mathscr{L}$, that $D\left(\mathscr{L}_{0}^{*}\right) \subset D\left(\mathscr{K}^{*}\right)$. Then $D\left(L_{0}^{*}\right)=$ $D\left(\left(\mathscr{L}_{0}+\mathscr{K}\right)^{*}\right)$.

Proof. Recall that $D\left(\left(\mathscr{L}_{0}+\mathscr{K}\right)^{*}\right)=\left\{f \in L^{2}(\mathscr{S}) \mid g \rightarrow\left(\left(\mathscr{L}_{0}+\mathscr{K}\right) g, f\right)\right.$ is continuous on $\left.D\left(\mathscr{L}_{0}+\mathscr{K}\right)\right\}$. If $f \in D\left(\mathscr{L}_{0}^{*}\right)$, then $\left(\left(\mathscr{L}_{0}+\mathscr{K}\right) g, f\right)=$ $\left(g,\left(\mathscr{L}_{0}^{*}+\mathscr{K}^{*}\right) f\right)$, so $D\left(\mathscr{L}_{0}^{*}\right) \subset D\left(\left(\mathscr{L}_{0}+\mathscr{K}\right)^{*}\right)$. Now by Lemma 2.iv dimension $\left(\left(\mathscr{L}_{0}+\mathscr{K}\right)^{*} \theta\left(\mathscr{L}_{0}+\mathscr{K}\right)\right)=$ dimension $\left(\mathscr{L}^{*} \theta \mathscr{L}_{0}\right)<\infty$. This forces $D\left(\mathscr{L}_{0}^{*}\right)=D\left(\left(\mathscr{L}_{0}+\mathscr{K}\right)^{*}\right)$.

In the regular case, that is when $\mathscr{J}=(a, b),-\infty<a<b<\infty$, the coefficients $a_{k}$ of $L$ are in $C^{k}([a, b])$ and $a_{n}(x) \neq 0$ for $x \in[a, b]$, the domain of $\mathscr{L}_{0}^{*}$ has an alternate description. Then $D\left(\mathscr{L}_{0}^{*}\right)$ is the set of all $f \in L^{2}(\mathscr{J})$ such that $f \in C^{n-1}([a, b]), f^{(n-1)}$ is absolutely continuous on $[a, b]$, and $L f \in L^{2}(\mathscr{J})$.

TheOREM 3.iv. Let $H=\mathscr{L}+\mathscr{K}, H_{0}=\mathscr{L}_{0}+\mathscr{K}$ as above. Suppose that for some $\mathfrak{l}_{0} \in \rho(H)$ we have dimension $\nu\left(H_{0}^{*}-\mathfrak{l}_{0} I\right)=n$. Then there are points $c_{i} \in \mathscr{F}, i=1, \cdots, n$, a neighborhood $U$ of $\mathfrak{l}_{0}$ and $a$ basis $t_{1}(x, \mathfrak{l}), \cdots, t_{n}(x, \mathfrak{l})$ for $\nu\left(H_{0}^{*}-\mathfrak{l} I\right)$ such that $t_{i}(\mathfrak{l})$ is analytic from $U \rightarrow L^{2}(\mathscr{F})$ and $t_{i}\left(c_{j}, \mathfrak{l}\right)=\delta_{i j}$ for $\mathfrak{l} \in U$. Moreover for fixed $x \in$ $\mathscr{F}, t_{i}(x, \mathfrak{l})$ is an analytic function. If $\sigma(H)$ is discrete we can choose $U=C \backslash\{$ closed countable set $\}$.

Proof. We start with the basis $s_{1}(x, \mathfrak{l}), \cdots, s_{n}(x, \mathfrak{l})$ constructed in (3.i). First we need to establish the analyticity of $s_{i}\left(c_{i}, \mathfrak{l}\right), c_{i} \in \mathscr{J}$. Letting $\mathfrak{l}_{1} \in \rho(H)$ we have 


$$
\begin{aligned}
\lim _{\mathfrak{i} \rightarrow \mathfrak{l}_{1}} \frac{s_{i}\left(c_{i}, \mathfrak{l}\right)-s_{i}\left(c_{i}, \mathfrak{l}_{1}\right)}{\mathfrak{l}-\mathfrak{I}_{1}} & =\lim _{\mathfrak{l} \rightarrow \mathfrak{1}_{1}} \frac{\left\{\left(I+\left(\mathfrak{l}-\mathfrak{I}_{1}\right) R_{H}(\mathfrak{l})\right] s_{i}\left(\mathfrak{l}_{i}\right)\right\}\left(c_{i}\right)-s_{i}\left(c_{i}, \mathfrak{l}_{1}\right)}{\mathfrak{l}-\mathfrak{I}_{1}} \\
& =\lim _{\mathfrak{l} \rightarrow 1_{1}}\left[R_{H}(\mathfrak{l}) s_{i}\left(\mathfrak{I}_{1}\right)\right]\left(c_{i}\right)=\left[R_{H}\left(\mathfrak{l}_{1}\right) s_{i}\left(\mathfrak{l}_{1}\right)\right]\left(c_{i}\right) .
\end{aligned}
$$

Here we have used the continuity of evaluation on $D(H)$ with the graph topology and the analyticity of $R_{H}(\mathfrak{l})$.

Now pick $c_{1} \in \mathscr{I}$ such that $s_{1}\left(c_{1}, \mathfrak{I}_{0}\right) \neq 0$ and define a basis for $\left(H_{0}^{*}-\mathfrak{l} I\right)$ by setting

$$
\begin{aligned}
& s_{1,1}(x, \mathfrak{l})=\frac{s_{1}(x, \mathfrak{l})}{s_{1}\left(c_{1}, \mathfrak{l}\right)} \\
& s_{i, 1}(x, \mathfrak{l})=s_{i}(x, \mathfrak{l})-\frac{s_{i}\left(c_{1}, \mathfrak{l}\right)}{s_{1}\left(c_{1}, \mathfrak{l}\right)} s_{1}(x, \mathfrak{l}), \quad i=2, \cdots, n .
\end{aligned}
$$

This basis is well defined and analytic if $s_{1}\left(c_{1}, \mathfrak{l}\right) \neq 0$, which, since $s_{1}\left(c_{1}, \mathfrak{l}\right)$ is analytic, will be everywhere in $\rho(H)$ except for a set of isolated points. Notice that $s_{1,1}\left(c_{1}, \mathfrak{l}\right)=1$ while $s_{i, 1}\left(c_{1}, \mathfrak{l}\right)=0, i=2, \cdots, n$.

Inductively define $s_{i, j}(x, \mathfrak{l})$ for $j=2, \cdots, n$ by

$$
\begin{aligned}
& s_{i, j}(x, \mathfrak{l})=s_{i, j-1}(x, \mathfrak{l}) \quad \text { if } i \leqq j-1 \\
& s_{j, j}(x, \mathfrak{l})=\frac{s_{j, j-1}(x, \mathfrak{l})}{s_{j, j-1}\left(c_{j}, \mathfrak{l}\right)} \\
& s_{i, j}(x, \mathfrak{l})=s_{i, j-1}(x, \mathfrak{l})-\frac{s_{i, j=1}\left(c_{j}, \mathfrak{l}\right)}{s_{j, j}\left(c_{j}, \mathfrak{l}\right)} s_{j, j}(x, \mathfrak{l}) \text { for } i>j,
\end{aligned}
$$

where $c_{j}$ is chosen so that $s_{j, j-1}\left(c_{j}, \mathfrak{Y}_{0}\right) \neq 0$. Then $\left\{s_{i, n}\right\}_{i=1}^{n}$ is a basis for $\nu\left(H_{0}^{*}-Y I\right)$ in an open set containing $\mathfrak{I}_{1}$ and including those points in $\rho(H)$ where $s_{i, i}\left(c_{i}, \mathfrak{l}\right) \neq 0, i=1, \cdots, n$. By construction we see that if $\sigma(H)$ is discrete, then the set where our new basis is not defined, which we call the singular set, must be countable and closed.

Moreover the collection $\left\{s_{i, n}(x, \mathfrak{l})\right\}_{i=1}^{n}$ satisfies $s_{j, n}\left(c_{i}, \mathfrak{l}\right)=0$ if $i<j$ and $s_{j, j}\left(c_{j}, \mathfrak{l}\right)=1, j=1, \cdots, n$. Without adding to the singular set we construct a new basis as follows: Define $t_{n}(x, \mathfrak{l})=s_{n, n}(x, \mathfrak{l})$, and inductively define $t_{m}(x, \mathfrak{l})=s_{m, n}(x, \mathfrak{l})-\sum_{k>m} s_{m, n}\left(c_{k}, \mathfrak{l}\right) t_{k}(x, \mathfrak{l})$ for $m=$ $(n-1),(n-2), \cdots, 1$. Then $\left\{t_{m}(x, \mathfrak{l})\right\}_{m=1}^{n}$ is the desired basis for $\boldsymbol{\nu}\left(H_{0}^{*}-\mathfrak{l} I\right)$.

To develop eigenfunction expansion for $\mathscr{L}+\mathscr{K}$ as above we use the notion of generalized resolvent. Let $\mathscr{H}$ be a subspace of the Hilbert space $\mathscr{G}$, let $S$ be a symmetric operator in $\mathscr{H}$, and suppose that $M$ is a self-adjoint extension of $S$ in $\mathscr{G}$. Then the generalized resolvent $R_{M}(\mathfrak{I}): \mathscr{H} \rightarrow \mathscr{H}$ is defined by $R_{M}(\mathfrak{l}) f=P(M-\mathfrak{l} I)^{-1} f$, $\mathfrak{l} \in C_{0}$, where $P$ is the orthogonal projection from $\mathscr{G}$ onto $\mathscr{H}$. We will need Theorem 4 of Coddington [5], which treats in addition the generalization of generalized resolvents to subspaces. 
THEOREM 3.v (Coddington). The generalized resolvent $R_{M}(\mathfrak{l})$ of a symmetric subspace in $\mathscr{H} \oplus \mathscr{C}$ corresponding to the self-adjoint extension $H$ in $\mathscr{G} \oplus \mathscr{G}, \mathscr{H} \subset \mathscr{G}$, satisfies:

(i ) $R_{M}(\mathfrak{l})$ exists as an operator on all of $\mathscr{L}$,

(ii) if $T(\mathfrak{l})=\left\{\left\{R_{M}(\mathfrak{l}) h, \mathfrak{l} R_{M}(\mathfrak{l}) h+h\right\} \mid h \in \mathscr{H}\right\}$, then $S \subset T(\mathfrak{l}) \subset S^{*}$,

(iii) $\left\|R_{M}(\mathfrak{l})\right\| \leqq 1 /(\|\operatorname{Im} \mathfrak{l}\|)$,

(iv) $R_{M}(\mathfrak{l})^{*}=R_{M}(\overline{\mathfrak{l}})$,

(v) $\operatorname{Im}\left(R_{M}(\mathfrak{l}) h, h\right) / \operatorname{Im} \mathfrak{l} \geqq\left\|R_{M}(\mathfrak{l}) h\right\|^{2}, h \in \mathscr{\mathscr { C }}$,

(vi) $R_{M}(\mathfrak{l})$ is analytic for $\operatorname{Im} \mathfrak{l} \neq 0$ in the uniform topology.

For the moment (ii) is of paramount importance. Suppose $H_{0}=$ $\mathscr{L}_{0}+\mathscr{K}$ as in (3.iv), and suppose $H_{0}$ has a self-adjoint extension $H$ in $L^{2}(I)$. Let $M$ be an extension of $H_{0}$ in a possibly larger space with generalized resolvent $R_{M}(\mathfrak{l})$. We want to understand $R_{H}(\mathfrak{l})-R_{M}(\mathfrak{l})$. If $f \in L^{2}(\mathscr{I})$ we have $\left\{R_{M}(\mathfrak{l}) f, \mathfrak{l} R_{M}(\mathfrak{l}) f+f\right\} \in H_{0}^{*}$ and $\left\{R_{H}(\mathfrak{l}) f, l R_{H}(\mathfrak{l}) f+\right.$ $f\} \in H_{0}^{*}$, so letting $A(\mathfrak{l}) f=R_{M}(\mathfrak{l}) f-R_{H}(\mathfrak{l}) f$ we have $\{A(\mathfrak{l}) f, \mathfrak{l} A(\mathfrak{l}) f\} \in H_{0}^{*}$, or $A(\mathfrak{l}) f \in \mathcal{\nu}\left(H_{0}^{*}-\mathfrak{l} I\right)$ for all $f \in L^{2}(\mathscr{I})$. Conclusions (i), (iv) and (vi) of (3.v) apply immediately to $A(\mathfrak{l})$, and (iii) becomes $\|A(\mathfrak{l})\| \leqq 2 /(|\operatorname{Im} \mathfrak{l}|)$.

Adopting the vector notation in [5], we let the components of $\alpha(\mathfrak{l})$ be an orthonormal basis for $\nu\left(H_{0}^{*}-\mathfrak{l} I\right), \mathfrak{l} \in C_{0}$. For matrices with the same number of rows and entries in $L^{2}(\mathscr{I})$ we define $(F, G)=$ $\int_{\mathscr{F}} G^{*} F$. It is easy to show that

$$
A(\mathfrak{l}) f=\alpha(\mathfrak{l})(A(\mathfrak{l}) f, \alpha(\mathfrak{l}))=\alpha(\mathfrak{l}) \alpha(\mathfrak{l})(f, \alpha(\overline{\mathfrak{l}}))
$$

where $a(\mathfrak{l})=(A(\mathfrak{l}) \alpha(\overline{\mathfrak{l}}), \alpha(\mathfrak{l}))$. Thus $A(\mathfrak{l})$ is a Hilbert-Schmidt integral operator and we can write $A(\mathfrak{l}) f=\int A(x, y, \mathfrak{l}) f(y) d y$ for all $f \in L^{2}(\mathscr{I})$, $\mathfrak{l} \in \boldsymbol{C}_{0}$.

If we use our analytic basis $\left\{t_{i}(x, \mathfrak{l})\right\}_{i=1}^{n}$ for $\nu\left(H_{0}^{*}-\mathfrak{l} I\right)$, developed in (3.iv), then except at the set of singular points we can write $A(x, y, \mathfrak{l})=t(x, \mathfrak{l}) \beta(\mathfrak{l}) t^{*}(y, \bar{l}) \quad$ where $t(x, \mathfrak{l})=\left(t_{1}(x, \mathfrak{l}), \cdots, t_{n}(x, \mathfrak{l})\right) . \quad$ Following the reasoning in ([5], p. 19) we get the following:

Lemma 3.vi. For those $\mathfrak{l} \in \boldsymbol{C}_{0}$ where $t(x, \mathfrak{l}), t^{*}(y, \overline{\mathfrak{l}})$ are defined we have $\beta(\mathfrak{l})$ analytic and $(\beta(\mathfrak{l}))^{*}=\beta(\overline{\mathfrak{l}})$.

Proof. For $f, g \in L^{2}(\mathscr{I})$ we have

$$
(A(\mathfrak{l}) f, g)=(t(\mathfrak{l}), g) \beta(\mathfrak{l})(f, t(\overline{\mathfrak{l}})) .
$$

Thus for $\mathfrak{I}_{0}$ outside the singular set we have

$$
\left(A(\mathfrak{l}) t\left(\mathfrak{I}_{0}\right), t\left(\overline{\mathfrak{I}}_{0}\right)\right)=\left(t(\mathfrak{l}), t\left(\mathfrak{I}_{0}\right)\right) \beta(\mathfrak{l})\left(t\left(\overline{\mathfrak{I}}_{0}\right), t(\overline{\mathfrak{I}})\right),
$$

where we use matrix inner product. Now for $\mathfrak{l}=\mathfrak{l}_{0}$ the matrices 
$\left(t(\mathfrak{l}), t\left(\mathfrak{l}_{0}\right)\right)$ and $\left(t\left(\overline{\mathfrak{l}}_{0}\right), t(\overline{\mathfrak{l}})\right)$ are invertible since the components of $t\left(\mathfrak{I}_{0}\right), t\left(\overline{\mathfrak{I}}_{0}\right)$ are linearly independent. Since these inner products are analytic for $\mathfrak{l}$ near $\mathfrak{l}_{0}$ the inverses exist and are analytic. Thus $\beta(\mathfrak{l})$ is analytic for $\mathfrak{l}$ near $\mathfrak{l}_{0}$, and since $\mathfrak{l}_{0}$ was arbitrary we have the first part of the result. For the symmetry $(\beta(\mathfrak{l}))^{*}=\beta(\overline{\mathfrak{l}})$ we use the fact that $A(\mathfrak{l})^{*}=$ $A(\overline{\mathfrak{l}})$, hence $\bar{A}(x, y, \mathfrak{l})=A(y, x, \overline{\mathfrak{l}})$. Now

$$
\overline{\beta_{i j}(\mathfrak{l})}=\bar{A}\left(c_{i}, c_{j}, \mathfrak{l}\right)=A\left(c_{j}, c_{i}, \overline{\mathfrak{l}}\right)=\beta_{j},{ }_{\imath}(\overline{\mathfrak{l}}),
$$

completing the proof.

IV. The kernel of $R_{H}(l)$. We turn to the nature of $R_{H}(\mathfrak{l})$ itself, where $H=\mathscr{L}+\mathscr{K}$ as above. In particular we show that if the order of $\mathscr{L}$ is at least two, then $R_{H}(\mathfrak{l})$ is integral operator with kernel $R_{H}(x, y, \mathfrak{l})$ continuous in the pair $(x, y)$ for $\mathfrak{l}$ fixed and analytic in $\mathfrak{l}$ for $(x, y)$ fixed.

Lemma 4.i. Suppose $G$ is an integral operator on $L^{2}(\mathscr{J}), G f=$ $\int_{G} G(x, y) f(y) d y$, such that mapping $x \rightarrow G(x, \cdot)$ is continuous from $\mathscr{F} \rightarrow L^{2}(\mathscr{F})$. Let $A$ be bounded. Then $G A$ is also an integral operator with kernel continuous in $L^{2}(\mathscr{T})$ as a function of $x$.

Proof. We have

$$
\begin{aligned}
(G A f)(x) & =\int_{\mathscr{S}} G(x, y)(A f)(y) d y=(A f, \bar{G}(x, \cdot)) \\
& =\left(f, A^{*} \bar{G}(x, \cdot)\right)=\int_{\mathscr{S}}\left[A^{*} \bar{G}(x, \cdot)\right](y) f(y) d y .
\end{aligned}
$$

Thus the kernel for $G A$ is $\overline{\left[A^{*} \bar{G}(x, \cdot)\right]}(y)$. The boundedness of $A$ yields the continuity.

Lemma 4.ii. Let $G$ and $K$ be bounded integral operators with kernels $G(x, y), K(y, z)$ respectively. If $x \rightarrow G(x, \cdot)$ and $z \rightarrow K(\cdot, z)$ are both continuous from $\mathscr{F} \rightarrow L^{2}(\mathcal{F})$, then $G K$ has the kernel $L(x, z)=\int_{\mathcal{f}} G(x, y) K(y, z) d y$, which is continuous in the pair $(x, z)$.

Proof.

$$
\begin{aligned}
\left|L\left(x_{1}, z_{1}\right)-L\left(x_{2}, z_{2}\right)\right|= & \mid \int_{\zeta}\left[G\left(x_{1}, y\right)-G\left(x_{2}, y\right)\right] K\left(y, z_{1}\right) \\
& +G\left(x_{2}, y\right)\left[K\left(y, z_{1}\right)-K\left(y, z_{2}\right)\right] d y \mid \\
\leqq & \left\|G\left(x_{1}, \cdot\right)-G\left(x_{2}, \cdot\right)\right\|\left\|K\left(\cdot, z_{1}\right)\right\| \\
& +\left\|G\left(x_{2}, \cdot\right)\right\|\left\|K\left(\cdot, z_{1}\right)-K(\cdot, z)\right\| .
\end{aligned}
$$


The result follows immediately from the right hand side of the inequality and our hypotheses.

LEMMA 4.iii. If $H$ is a self-adjoint operator and $A$ is a symmetric operator which is H-bounded with $H$-bound less than 1 , then for $\mathfrak{l} \in \boldsymbol{C}$

$$
R_{H-A}(\mathfrak{l})=R_{H}(\mathfrak{l})+R_{H}(\mathfrak{l}) A\left[I+R_{H-A}(\mathfrak{l}) A\right] R_{H}(\mathfrak{l}) .
$$

Proof. By Theorem 2.i $H-A$ is self-adjoint on $D(H)$. Since $\left[I-R_{H}(\mathfrak{l}) A\right] R_{H-A}(\mathfrak{l})=R_{H}(\mathfrak{l})$ we have

$$
R_{H-A}(\mathfrak{l})=R_{H}(\mathfrak{l})+R_{H}(\mathfrak{l}) A R_{A-H}(\mathfrak{l}) .
$$

Thus

$$
\begin{aligned}
R_{H-A}(\mathfrak{l}) A & =R_{H}(\mathfrak{l}) A+R_{H}(\mathfrak{l}) A R_{H-A}(\mathfrak{l}) A \\
& =R_{H}(\mathfrak{l}) A\left[I+R_{H-A}(\mathfrak{l}) A\right] .
\end{aligned}
$$

Consequently

$$
\begin{aligned}
I+R_{H-A}(\mathfrak{l}) A & =R_{H-A}(\mathfrak{l})(H-\mathfrak{l} I) \\
& =I+R_{H}(\mathfrak{l}) A\left[I+R_{H-A}(\mathfrak{l}) A\right] .
\end{aligned}
$$

Applying $R_{H}(\mathfrak{l})$ to the right gives the result.

THEOREM 4.iv. Let $H$ be self-adjoint in $L^{2}(\mathscr{I}), A$ symmetric and bounded. Suppose for $\mathfrak{l} \in C_{0}$ the operator $R_{H}(\mathfrak{l})$ is an integral operator with a kernel $R_{H}(x, y, \mathfrak{l})$ that is continuous in $(x, y)$. If the mapping $x \rightarrow R_{H}(x, \cdot, \mathfrak{l})$ is continuous from $\mathscr{I} \rightarrow L^{2}(\mathscr{I})$, then $R_{H-A}(\mathfrak{l})$ is also an integral operator with kernel continuous in $(x, y)$.

Proof. Because $R_{H}(x, y, \mathfrak{l})=\overline{R_{H}}(y, x, \overline{\mathfrak{l}})$ we see that the mapping $y \rightarrow R_{H}(\cdot, y, \mathfrak{l})$ is continuous from $\mathscr{F} \rightarrow L^{2}(\mathscr{I})$. By 4 .iii we have

$$
R_{H-A}(\mathfrak{l})=R_{H}(\mathfrak{l})+R_{H}(\mathfrak{l}) A\left[I+R_{H-A}(\mathfrak{l}) A\right] R_{H}(\mathfrak{l}),
$$

and $R_{H}(\mathfrak{l})$ has continuous kernel.

Now Lemma $4 . \mathrm{i}$ shows that both $R_{H}(\mathfrak{l}) A$ and $R_{H}(\mathfrak{l}) A R_{H-A}(\mathfrak{l}) A$ are integral operators with kernels $G_{i}(x, y, \mathfrak{l}), i=1,2$, such that the mappings $x \rightarrow G_{i}(x, \cdot, \mathfrak{l})$ are continuous from $\mathscr{I} \rightarrow L^{2}(\mathscr{F})$. Finally Lemma 4.ii finishes the proof.

THEOREM 4.v. Let $\mathscr{L}$ be a self-adjoint ordinary differential operator on $L^{2}(a, b)$ agreeing with $L=\sum_{k=0}^{n} a_{k}(t) D^{k}$ on its domain. Then for $\mathfrak{l} \in C_{0}$ the resolvent $R_{L}(\mathfrak{l})$ is an integral operator with kernel $R_{\mathscr{L}}(x, y, \mathfrak{l})$ such that the mapping $x \rightarrow R_{\mathscr{L}}(x, \cdot, \mathfrak{l})$ is continuous from $(a, b) \rightarrow L^{2}(a, b)$. 
Proof. From Coddington [2], [5], we know that $R_{L}(\mathfrak{l})$ is an integral operator of Carleman type whose kernel is, for fixed $\mathfrak{l}$, continuous in $(x, y)$ for $x \neq y$ and which, for fixed $y$, satisfies $L f=$ $\mathfrak{l} f$ as long as $x \neq y$. Moreover the kernel extends continuously to the diagonal $x=y$ from either above or below if $n=1$, and is continuous in case $n \geqq 2$.

Suppose that $a<x_{1}<x_{2}<b$. Then

$$
\begin{aligned}
\int_{a}^{b} \mid R_{\mathscr{L}}\left(x_{1}, y, \mathfrak{l}\right)- & \left.R_{\mathscr{L}}\left(x_{2}, y, \mathfrak{l}\right)\right|^{2} d y=\int_{a}^{x_{1}}\left|R_{\mathscr{L}}\left(x_{1}, y, \mathfrak{l}\right)-R_{\mathscr{S}}\left(x_{2}, y, \mathfrak{l}\right)\right|^{2} d y \\
& +\int_{x_{1}}^{x_{2}}\left|R_{\mathscr{L}}\left(x_{1}, y, \mathfrak{l}\right)-R_{\mathscr{L}}\left(x_{2}, y, \mathfrak{l}\right)\right|^{2} d y \\
& +\int_{x_{2}}^{b}\left|R_{\mathscr{C}}\left(x_{1}, y, \mathfrak{l}\right)-R_{\mathscr{H}}\left(x_{2}, y, \mathfrak{l}\right)\right|^{2} d y .
\end{aligned}
$$

Since $R_{\mathscr{G}}(x, y, \mathfrak{l})$ is, for fixed $\mathfrak{l}$, uniformly bounded on compact subsets of $(a, b) \times(a, b)$, we see that

$$
\lim _{x_{1} \rightarrow x_{2}} \int_{x_{1}}^{x_{2}}\left|R_{\mathscr{f}}\left(x_{1}, y, \mathfrak{l}\right)-R_{\mathscr{L}}\left(x_{2}, y, \mathfrak{l}\right)\right|^{2} d y=0 .
$$

Now consider the $n$-dimensional vector space of solutions to $L f=\mathfrak{l} f$. This vector space has a subspace of dimension $m$ whose elements, when suitably restricted, are in $L^{2}\left(x_{2}, b\right)$. Let a basis for this subspace be given by $\phi_{1}(y), \cdots, \phi_{m}(y)$. Since, for $x$ and $\mathfrak{l}$ fixed, we have $R_{\mathscr{C}}(x, y, \mathfrak{l}) \in L^{2}(a, b)$, we can write for $\mathfrak{l}$ fixed and $y \in\left[x_{2}, b\right)$, $R_{\mathscr{f}}(x, y, \mathfrak{l})=\sum_{i=1}^{m} \alpha_{i}(x) \phi_{i}(y)$. We claim that as $x_{1} \rightarrow x_{2}, \alpha_{i}\left(x_{1}\right) \rightarrow \alpha_{i}\left(x_{2}\right)$.

To see this we use the continuity of $R_{\mathscr{f}}(x, y, \mathfrak{l})$ in $(x, y)$. We know that for $y$ in any compact interval of $\left[x_{2}, b\right)$ we have

$$
\lim _{x_{1} \rightarrow x_{2}}\left|\Sigma\left\{\alpha_{i}\left(x_{1}\right) \phi_{i}(y)-\alpha_{i}\left(x_{2}\right) \phi_{i}(y)\right\}\right|=0
$$

uniformly in $y$. By the linear independence of $\phi_{i}(y)$ on any interval the coefficients must be converging. That is $\lim _{x_{1} \rightarrow x_{2}}\left[\alpha_{i}\left(x_{1}\right)-\alpha_{i}\left(x_{2}\right)\right]=0$.

Since $\phi_{i} \in L^{2}\left(x_{2}, b\right)$ we have

$$
\lim _{x_{1} \rightarrow x_{2}} \int_{x_{2}}^{b}\left|R_{\mathscr{E}}\left(x_{1}, y, \mathfrak{l}\right)-R_{\mathscr{C}}\left(x_{2}, y, \mathfrak{l}\right)\right|^{2} d y=0 .
$$

Using a similar argument of the integral from $a$ to $x_{1}$ gives the result.

THEOREM 4.vi. Let $H$ be a self-adjoint operator on $L^{2}(\mathscr{J})$ and suppose $\mathfrak{l} \in \rho(H)$. If $R_{H}(\mathfrak{l})$ is an integral operator with kernel $R_{H}(x, y, \mathfrak{l})$ continuous as a function of $(x, y)$, then $R_{H}(x, y, \mathfrak{l})$ is analytic for $(x, y)$ fixed. 
Proof. From the resolvent equation we have

$$
R_{H}\left(x, y, \mathfrak{l}_{1}\right)-R_{H}\left(x, y, \mathfrak{l}_{2}\right)=\left(\mathfrak{l}_{1}-\mathfrak{I}_{2}\right) \int R_{H}\left(x, z, \mathfrak{I}_{1}\right) R_{H}\left(z, y, \mathfrak{I}_{2}\right) d z
$$

for all $x, y \in \mathscr{F}, \mathfrak{l}_{1}$ and $\mathfrak{I}_{2}$ in $\rho(H)$. Thus $R_{H}(x, y, \mathfrak{l})$ will be analytic if we can show the existence of $\lim _{\mathfrak{l}_{1} \rightarrow \mathfrak{I}_{2}} \int R_{H}\left(x, z, \mathfrak{I}_{1}\right) R_{H}\left(z, y, \mathfrak{I}_{2}\right) d z$. In other words, it suffices to show that, for $x$ fixed, the map $\mathfrak{l} \rightarrow$ $R_{H}(x, \cdot, \mathfrak{l})$ is continuous from $\rho(H) \rightarrow L^{2}(\mathscr{F})$.

The argument runs as follows:

$$
\begin{aligned}
\left\|R_{H}\left(x, \cdot, \mathfrak{l}_{1}\right)-R_{H}\left(x, \cdot, \mathfrak{l}_{2}\right)\right\|^{2}= & \int\left|R_{H}\left(x, y, \mathfrak{l}_{1}\right)-R_{H}\left(x, y, \mathfrak{l}_{2}\right)\right|^{2} d y \\
= & \left(R_{H}\left(\mathfrak{l}_{1}\right)-R_{H}\left(\mathfrak{l}_{2}\right)\right)\left(\overline{R_{H}}\left(x, \cdot, \mathfrak{l}_{1}\right)-\overline{R_{H}}\left(x, \cdot, \mathfrak{l}_{2}\right)\right) \\
\leqq & \left\|R_{H}\left(\mathfrak{l}_{1}\right)-R_{H}\left(\mathfrak{l}_{2}\right)\right\| \| R_{H}\left(x, \cdot, \mathfrak{l}_{1}\right) \\
& -R_{H}\left(x, \cdot \mathfrak{l}_{2}\right) \| .
\end{aligned}
$$

Thus $\left\|R_{H}\left(x, \cdot, \mathfrak{l}_{1}\right)-R_{H}\left(x, \cdot, \mathfrak{l}_{2}\right)\right\| \leqq\left\|R_{H}\left(\mathfrak{l}_{1}\right)-R_{H}\left(\mathfrak{l}_{2}\right)\right\|$. Since $R_{H}(\mathfrak{l})$ is analytic in the uniform topology this implies the continuity of the map $\mathfrak{l} \rightarrow R_{I I}(x, \cdot, \mathfrak{l})$ from $\rho(H) \rightarrow L^{2}(\mathscr{I})$ for each fixed $x$.

V. Spectral measures. Returning to the operators $\mathscr{L}+\mathscr{K}$ described in the introduction, we observe that since the kernel $K(x, y)$ for $\mathscr{K}$ is compactly supported in $\mathscr{I} \times \mathscr{I}$, we can find a compact square $B \times B \subset \mathscr{J} \times \mathscr{J}$ off which $K(x, y)$ vanishes, and an open interval $\mathscr{J}$ such that $B \subset \mathscr{J}$ and the closure of $\mathscr{J}$ is compact in $\mathscr{F}$. Now $L^{2}(\mathscr{I})$ decomposes as an orthogonal sum $L^{2}(\mathscr{J}) \oplus L^{2}(\mathscr{I} \backslash \mathscr{J})$. Define a symmetric operator by restricting $H=\mathscr{L}+\mathscr{K}$ to $C_{0}^{\infty}(\mathscr{J})$, then closing the operator $S_{\mathscr{J}}$ in $L^{2}(\mathscr{J})$. By virtue of 2.i, 2.iii and 2.iv, $D\left(S_{\mathscr{f}}\right)$ is compactly embedded in $L^{2}(\mathscr{J})$ and dimension $\left(S_{\mathscr{f}}^{*} \theta S_{\mathscr{f}}\right)<\infty$. Observe that $H$ is a self-adjoint extension of $S_{\mathcal{F}}$ in the larger space $L^{2}(\mathscr{J})$, where we are identifying $L^{2}(\mathscr{J})$ with $L^{2}(\mathscr{J}) \oplus\{0\} \subset L^{2}(\mathscr{J}) \oplus$ $L^{2}(\mathscr{F} \backslash \mathscr{J})$.

Using the results of $\S$ III we can find, for all $\mathfrak{l}$ such that $\nu\left(S_{\mathcal{F}}-\mathfrak{l} I\right)=0$, one of the bases which we previously denoted $\left\{t_{i}(x, \mathfrak{l})\right\}_{i=1}^{n}$. Since $\mathscr{J} \backslash B$ has interior and each function $t_{i}(x, \mathfrak{l})$ satisfies

$$
(L-\mathfrak{l}) t_{i}(x, \mathfrak{l})=0
$$

on $\mathscr{J} \backslash B$ for those $\mathfrak{l}$ for which it is defined, we can define natural extensions of $t_{i}(x, \mathfrak{l})$ to the interval $\mathscr{F}$ by continuing the solutions of the differential equation. More specifically, $\mathscr{I} \backslash B$ consists of two components, each of which has as a subset part of the interior of $\mathscr{J} \backslash B$. Call these components $C_{1}$ and $C_{2}$. On $C_{1}$ define an extension of $t_{i}(x, \mathfrak{l})$ as the unique solution of $(L-\mathfrak{l}) f=0$ which agrees with 
$t_{i}(x, \mathfrak{l})$ on $C_{1} \cap(\mathscr{J} \backslash B)$. Defining an extension on $C_{2}$ analogously, we will henceforth call this extension $t_{i}(x, \mathfrak{l})$. The analyticity of these functions is preserved when they are extended.

Lemma 5.i. Let $L$ be a formal differential operator of order $n$ defined on an open interval $\mathscr{F}$. Suppose $\mathscr{J}=(a, b)$ is an open subinterval of $\mathscr{F}$ and $t(x, \mathfrak{l})=\left(t_{1}(x, \mathfrak{l}), \cdots, t_{n}(x, \mathfrak{l})\right)$ is a basis for the solutions of $(L-\mathfrak{l} I) f(x, \mathfrak{l})=0$ on $\mathscr{J}$. If the mapping $\mathfrak{l} \rightarrow t_{i}(\mathfrak{l})$ is, for each $i$, analytic from $U_{\text {open }} \rightarrow L^{2}(\mathscr{J})$, then for each $y \in \mathscr{J}, b<y$, the extension of $t_{i}(x, \mathfrak{l})$ satisfying $(L-\mathfrak{l} I) t_{i}(x, \mathfrak{l})=0$ for $x \in(a, y)$ also has $\mathfrak{l} \rightarrow t_{i}(x, \mathfrak{l})$ analytic from $U \rightarrow L^{2}(a, y)$.

Proof. Let $s(x, \mathfrak{l})=\left(s_{1}(x, \mathfrak{l}), \cdots, s_{n}(x, \mathfrak{l})\right)$ be the solution of $(L-\mathfrak{l}) s(x, \mathfrak{l})=0$ satisfying $s_{i}^{(j-1)}(c, \mathfrak{l})=\delta_{i j}$, for some $c \in \mathscr{F}_{\text {. }}$ Then for each $\mathfrak{l} \in U$ the extension of the solution $t(x, \mathfrak{l})$ to $\mathscr{J}$ must satisfy $t(x, \mathfrak{l})=s(x, \mathfrak{l}) D(\mathfrak{l})$ for some $n \times n$ matrix $D(\mathfrak{l})$. Since $\mathfrak{l} \rightarrow s_{i}(x, \mathfrak{l})$ is analytic from $C \rightarrow L^{2}[(a+b) / 2, y]$, it suffices to show that $\mathfrak{l} \rightarrow D(\mathfrak{l})$ is analytic for $\mathfrak{l} \in U$.

Fix $\mathfrak{l}_{0} \in U$. Since the components of $s(x, \mathfrak{l})$ are linearly independent in $L^{2}[(a+b) / 2, b]$, we have that $S(\mathfrak{l})=\int_{(a+2) / 2}^{b} s^{*}\left(x, \mathfrak{l}_{0}\right) s(x, \mathfrak{l}) d x$ is analytic and invertible for $\mathfrak{I}$ near $\mathfrak{I}_{0}$. Moreover by the assumption of analyticity of $t(x, \mathfrak{l})$ in $L^{2}(\mathscr{J})$ we see that $T(\mathfrak{l}) \mathrm{t}=\int_{(a+) / 2}^{b} s^{*}\left(x, \mathfrak{l}_{0}\right) t(x, \mathfrak{l}) d x$ is analytic for $\mathfrak{l}$ near $\mathfrak{l}_{0}$. Since $T(\mathfrak{l})=S(\mathfrak{l}) D(\mathfrak{l})$ we have $D(\mathfrak{l})=$ $S^{-1}(\mathfrak{l}) T(\mathfrak{l})$, which shows that $\mathfrak{l} \rightarrow D(\mathfrak{l})$ is analytic for $\mathfrak{l} \in U$.

Lemma 5.ii. Let $\mathscr{J}$ be as above. Then 1 , the set of eigenvalues for $S_{\mathscr{f}}$, is independent of $\mathscr{J}$.

Proof. Let $\lambda$ be an eigenvalue for $S_{\mathcal{F}}$ with eigenfunction $\phi_{\lambda}$ for some interval $\mathscr{J}$. Then by the uniqueness of solutions to the initial value problem for ordinary differential equations $\phi_{2}$ is supported in $B$. Consequently $\phi_{\lambda}$ is an eigenfunction for one $S_{\mathscr{J}}$ if and only if it is an eigenfunction for all $S_{\mathscr{f}}$.

Lemma 5.iii. Let $\mathcal{J}$ be as above and let $\widetilde{J}$ be an open interval containing $\mathscr{J}$ such that the closure of $\widetilde{J}$ is compact in $\mathscr{F}$. Suppose that $S_{\tilde{J}}$ is the minimal symmetric operator associated with $H$ and $\widetilde{J}$. Then the extended functions $\left\{t_{i}(x, \mathfrak{l})\right\}_{i=1}^{n}$, when restricted to $\widetilde{J}$, form a basis for $\nu\left(S_{\widetilde{J}}^{*}-\mathfrak{l} I\right)$ for those $\mathfrak{l} \in \boldsymbol{C}$ where $\left\{t_{i}(x, \mathfrak{l})\right\}_{i=1}$ is defined.

Proof. According to 2.iv the dimension of $S_{\widetilde{J}}^{*} \theta S_{\widetilde{J}}$ is the same as that of the corresponding difference for the minimal and maximal 
operators corresponding to $L$ on $L^{2}(\widetilde{J})$. This is just $2 n=2$ dimension $\boldsymbol{\nu}\left(S_{\tilde{J}}^{*}-\mathfrak{l} I\right), \mathfrak{l} \in \boldsymbol{C}_{0}$. On the other hand $\left\{t_{i}(x, \mathfrak{l})\right\}_{i=1}^{n}$ consists of linearly independent functions which, by their definition, satisfy the integrodifferential equation

$$
L t_{i}(x, \mathfrak{l})+\int_{\tilde{J}} K(x, y) t_{i}(y, \mathfrak{l}) d y=\mathfrak{l} t_{i}(x, \mathfrak{l}) .
$$

Since $t_{i}(x, \mathfrak{l})$ is the domain of the maximal operator for $L$, we have, by 3.iii, exhibited a basis for $\nu(\stackrel{*}{J}-\mathfrak{l} I)$.

At this point we will assume that the self-adjoint operator $\mathscr{L}$ has order $n \geqq 2$. In case $n=1$ slight modifications in the arguments will be necessary since $R_{\mathscr{L}}(x, y, \mathfrak{l})$ will not, in that case, be continuous as a function of $(x, y)$. In case $n \geqq 2$, Theorems $4 . v$ and 4 .vi guarantee that since $R_{\mathscr{L}}(\mathrm{l})$ is an integral operator of Carleman type with kernel $R_{\mathscr{L}}(x, y, \mathfrak{l})$ continuous in $(x, y)$, the operator $R_{H}(\mathfrak{l})$ shares this property. Define the $n \times n$ matrix-valued function $\psi(\mathfrak{l})=\left(\psi_{i j}(\mathfrak{l})\right)$ by $\psi_{i j}(\mathfrak{l})=$ $R_{H}\left(c_{i}, c_{j}, \mathfrak{l}\right)$, where $c_{i}$ is as in Theorem 3.iv.

THEOREM 5.iv. The $n \times n$ matrix function $\psi$ satisfies:

(i) $\psi$ is analytic in $C_{0}$,

(ii) $[\psi(\mathfrak{l})]^{*}=\psi(\overline{\mathfrak{l}})$,

(iii) $(\operatorname{Im} \psi(\mathfrak{l})) / \operatorname{Im} \mathfrak{I} \geqq 0, \operatorname{Im} \psi(\mathfrak{l})=\left(\psi(\mathfrak{l})-\psi^{*}(\mathfrak{l})\right) / 2 i$.

Proof. (i) Follows immediately from 4.vi.

(ii) Follows from $R_{H}\left(c_{i}, c_{j}, \mathfrak{l}\right)=\bar{R}_{H}\left(c_{j}, c_{i}, \mathfrak{l}\right)$.

(iii) We know from $3 . \mathrm{v}$ part $\mathbf{v}$ that $\left(\operatorname{Im}\left(R_{H}(\mathfrak{l}) h, h\right)\right) / \operatorname{Im} \mathfrak{l} \geqq 0$. Let $z_{1}, \cdots, z_{n}$ be any complex numbers, and $h_{m}(x)$ be smooth functions satisfying:

(a) Support of $h_{m}(x) \subset[-1 / m, 1 / m], m=1,2,3, \cdots$,

(b) $\int_{\mathcal{J}} h_{m}(x) d x=1, m=1,2,3, \cdots$

(c) $h_{m}(x) \geqq 0, m=1,2, \cdots$.

Let $k_{m}(x)=\sum_{i=1}^{n} z_{i} h_{m}\left(x-c_{i}\right)$. Then for each $m$ we have

$$
\begin{aligned}
& \frac{\operatorname{Im}\left(R_{H}(\mathfrak{l}) k_{m}, k_{m}\right)}{\operatorname{Im} \mathfrak{l}}=\frac{1}{\operatorname{Im} \mathfrak{l}} \iint\left[\frac{R_{H}(x, y, \mathfrak{l})-R_{H}(x, y, \overline{\mathfrak{l}})}{2 i}\right] \\
& k_{m}(x) \cdot \bar{k}_{m}(y) d x d y \geqq 0 .
\end{aligned}
$$

Since this last inequality is true for all $m$, and the kernels $R_{H}(x, y, \mathfrak{l}), R_{H}(x, y, \overline{\mathfrak{l}})$ are continuous in $(x, y)$ for $\mathfrak{l} \in \boldsymbol{C}_{0}$, we conclude that

$$
\sum_{i, j} z_{i} \bar{z}_{j} \frac{\operatorname{Im} R_{H}\left(c_{i}, c_{j}, \mathfrak{l}\right)}{\operatorname{Im} \mathfrak{l}}=\sum_{i, j=1}^{n} \frac{\operatorname{Im} \psi_{i j}(\mathfrak{l})}{\operatorname{Im} \mathfrak{l}} z_{i} \bar{z}_{j} \geqq 0,
$$


which is (iii).

THEOREM 5.v (Coddington). The matrix $\rho$ defined by $\rho(\lambda)=$ $\lim _{\varepsilon \rightarrow+0} 1 / \pi \int_{0}^{\lambda} \operatorname{Im} \psi(\nu+i \varepsilon) d \nu$ exists, is nondecreasing, and is of bounded variation on any finite interval.

Proof. This theorem, which appears in Coddington [3], depends only on the validity of 5.iv.

The function $\rho(\lambda)$ induces a matrix-valued measure in $R$. For a development of this idea see Dunford and Schwartz [7].

VI. Eigenfunction expansions. Fix an extended basis $\left\{t_{i}(x, \mathfrak{l})\right\}_{i=1}^{n}$ as defined at the beginning of section five, and let $\Delta$ be a compact interval which is contained in the set of $\mathfrak{l}$ in the domain of $t(x, \mathfrak{l})=$ $\left(t_{1}(x, \mathfrak{l}), \cdots, t_{n}(x, \mathfrak{l})\right)$. Observe that Theorems 3.iv and 2.vi guarantee that if $\lambda \notin \Lambda$, then there is a basis $\left\{t_{i}(x, \mathfrak{l})\right\}_{i=1}^{n}$ and an interval $\Delta$ as above with $\lambda \in \Delta$. Assume that the endpoints of $\Delta$ are continuity points for $E_{H}(\lambda)$, the resolution of the identity associated with $H=$ $\mathscr{L}+\mathscr{K}$.

Define an operator $R_{1}(\mathfrak{l})$ with kernel $R_{1}(x, y, \mathfrak{l})=t(x, \mathfrak{l}) \psi(\mathfrak{l}) t^{*}(y, \overline{\mathfrak{l}})$. Since $t_{i}(x, \mathfrak{l})$ may not be in $L^{2}(\mathscr{F})$ this kernel may not be of Carleman type, but we do know that each $t_{i}(x, \mathfrak{l})$ is continuous on every compact subinterval of $\mathscr{F}$. Our first goal is to prove that for $f, g \in C_{0}^{\infty}(\mathscr{F})$,

$$
\left(E_{I I}(\Delta) f, g\right)=\lim _{\varepsilon \rightarrow+0} \frac{1}{\pi} \int_{\Delta}\left(\operatorname{Im} R_{1}(\nu+i \varepsilon) f, g\right) d \nu \text {. }
$$

It is well known that

$$
\left(E_{H}(\Delta) f, g\right)=\lim _{\varepsilon \rightarrow+\uparrow} \frac{1}{\pi} \int_{\Delta}\left(\operatorname{Im} R_{H}(\nu+i \varepsilon) f, g\right) d \nu .
$$

Let $J$ be an interval with compact closure in $\mathscr{J}$ such that $J$ contains the union of the supports of $f$ and $g$. By 2.vi and the compactness of $\Delta$ there is a finite collection $\left\{H_{\alpha}\right\}$ of self-adjoint extensions of $S_{J}$ in $L^{2}(J)$ such that $\Delta \subset \bigcup_{\alpha} \rho\left(H_{\alpha}\right)$. Thus we can decompose $\Delta$ into a finite collection of closed intervals $\Delta_{\beta}$ with pairwise disjoint interiors such that $\Delta=\bigcup_{\beta} \Delta_{\beta}$ and $\Delta_{\beta} \subset \rho\left(H_{\alpha}\right)$ for some $\alpha$. Let $H_{\beta} \in\left\{H_{\alpha}\right\}$ satisfy $\Delta_{\beta} \subset \rho\left(H_{\beta}\right)$. Note that we may choose the intervals $\Delta_{\beta}$ so that their endpoints are continuity points for $E_{H}(\lambda)$ and $E_{H_{\beta}}(\lambda)$. We assume such a choice has been made.

For each $\beta$ define an operator $G_{\beta}(\mathfrak{l})$ with kernel

$$
G_{\beta}(x, y, \mathfrak{l})=R_{\beta}(x, y, \mathfrak{l})-\sum_{i, j=1}^{n} t_{i}(x, \mathfrak{l}) R_{\beta}\left(c_{i}, c_{j}, \mathfrak{l}\right) \bar{t}_{j}(y, \overline{\mathfrak{l}})
$$


where $R_{\beta}(x, y, \mathfrak{l})$ is the kernel of the resolvent of $H_{\beta}$. If $\mathfrak{l} \in \boldsymbol{C}_{0}$ and $x, y \in J$ we know that

$$
R_{H}(x, y, \mathfrak{l})-G_{\beta}(x, y, \mathfrak{l})=t(x, \mathfrak{l}) \Phi_{\beta}(\mathfrak{l}) t^{*}(y, \mathfrak{l}),
$$

where $\Phi_{\beta}$ is some $n \times n$ matrix-valued function. The reasoning here is exactly as that between 3.v and 3.vi. By construction $G_{\beta}\left(c_{i}, c_{j}, \mathfrak{l}\right)=$ 0 , so $\Phi_{\beta}=\left(R_{H}\left(c_{i}, c_{j}, \mathfrak{l}\right)\right)=\psi(\mathfrak{l})$. Consequently,

$$
\begin{aligned}
\left(E_{H}(\Delta) f, g\right)= & \lim _{\varepsilon \rightarrow+0} \frac{1}{\pi} \sum_{\beta} \int_{\Delta_{\beta}}\left(\operatorname{Im} R_{H}(\nu+i \varepsilon) f, g\right) d \nu \\
= & \lim _{\varepsilon \rightarrow+0} \frac{1}{\pi} \sum_{\beta} \int_{\Delta_{\beta}}\left(\operatorname{Im} G_{\beta}(\nu+i \varepsilon) f, g\right) d \nu \\
& +\lim _{\varepsilon \rightarrow+0} \frac{1}{\pi} \sum_{\beta} \int_{\Delta_{\beta}}\left(\operatorname{Im} R_{1}(\nu+i \varepsilon) f, g\right) d \nu .
\end{aligned}
$$

We claim that $\lim _{\varepsilon \rightarrow+0} \int_{\Delta_{\beta}}\left(\operatorname{Im} G_{\beta}(\nu+i \varepsilon) f, g\right) d \nu=0$. To see this we only need note that since $\Delta_{\beta} \subset \rho\left(H_{\beta}\right)$ the functions $R_{\beta}(x, y, \mathfrak{l})$ and $t(x, \mathfrak{l})$ are analytic in a neighborhood of $\Delta_{\beta}$, so our claim is a consequence of Cauchy's theorem and the definition of $G_{\beta}(\mathfrak{l})$.

We have now proved:

Lemma 6.i. For $f, g \in C_{0}^{\infty}(\mathscr{I}), \Delta$ a compact subinterval of $R$ where $t(x, \mathfrak{l})$ is defined, and assuming the endpoints of $\Delta$ are continuity points for $E_{H}(\lambda)$,

$$
\left(E_{H}(\Delta) f, g\right)=\lim _{\varepsilon \rightarrow+0} \frac{1}{\pi} \int_{\Delta}\left(\operatorname{Im} R_{1}(\nu+i \varepsilon) f, g\right) d \nu .
$$

Let $\Delta$ be as above, and recall that there is a matrix-valued measure $d \rho(\nu)$ defined on $R$, where $\rho(\nu)=\lim _{\varepsilon \rightarrow+0} 1 / \pi \int_{0}^{\nu} \operatorname{Im} \psi(\lambda+i \varepsilon) d \lambda$. If $f \in C_{0}^{\infty}(\mathscr{F})$ we will define a mapping $f \rightarrow \hat{f}$ from $L^{2}(\mathscr{F}) \rightarrow L^{2}(d \rho)$ which can be extended by continuity to all of $L^{2}(\mathscr{I})$.

Theorem 6.ii. Let $f \in C_{0}^{\infty}(\mathscr{F})$. Define $\hat{f}(\boldsymbol{\nu})$ by

$$
\hat{f}(\nu)=\int_{\mathscr{\Omega}} f(x) t^{*}(x, \nu) d x .
$$

Then

$$
E_{I I}(\Delta) f=\int_{\Delta} t(x, \nu) d \rho(\nu) \hat{f}(\nu)=\int_{\Delta} \sum_{j, k=1}^{n} t_{j}(x, \nu) \hat{f}_{k}(\nu) d \rho_{j k}(\nu) .
$$

Moreover, $\left[E_{H}(\Delta) f\right]^{\wedge}=\chi_{-} \hat{f}$, where $\chi_{\Delta}$ is the characteristic function of $\Delta$. 
Proof. By 6.i

$$
\begin{aligned}
\left(E_{H}(\Delta) f, g\right)= & \lim _{\varepsilon \rightarrow+0} \frac{1}{\pi} \int_{\Delta}\left(\operatorname{Im} R_{1}(\nu+i \varepsilon) f, g\right) d \nu \\
= & \lim _{\varepsilon \rightarrow+0} \frac{1}{2 \pi i} \int_{\Delta} \sum_{\Delta, k=1}^{n}\left\{\psi_{j_{k}}(\nu+i \varepsilon)\left(g, t_{j}(\nu-i \varepsilon)\right)\left(t_{k}(\nu+i \varepsilon), f\right)\right. \\
& \left.-\psi_{j_{k}}(\nu-i \varepsilon)\left(g, t_{j}(\nu+i \varepsilon)\right)\left(t_{k}(\nu-i \varepsilon), f\right)\right\} d \nu .
\end{aligned}
$$

Picking up the reasoning in Coddington [3] (following 4.2) we can evaluate the limits and get the first assertion of the theorem.

The second assertion, that the transform of $E_{H}(\Delta)$ is multiplication by the characteristic function of $\Delta$, is proved in Coddington and Dijksma [6] in their Theorem 6.5. It runs as follows. For $f, g \in$ $C_{0}^{\infty}(\mathscr{J})$ we have

$$
\left(E_{H}(\Delta) f, g\right)=\left(\widehat{E_{H}(\Delta) f}, \widehat{g}\right)=\left(\chi_{\Delta} \hat{f}, g\right)
$$

where this last inner product is in $L^{2}(d \rho)$. Thus $\left[E_{H}(\Delta) f\right]^{\wedge}=\chi_{\Delta} \hat{f}+\zeta$ where $\zeta$ is orthogonal to the image of $L^{2}(\mathscr{I})$ under the mapping $\uparrow$. But

$$
\left\|\left[E_{H}(\Delta) f\right]^{\wedge}\right\|^{2}=\left(E_{H}(\Delta) f, f\right)=\left(\chi_{\iota} \hat{f}, \hat{f}\right)=\left\|\chi_{\Delta} \hat{f}\right\|^{2}
$$

implies that $\zeta=0$.

There are now two ways in which we can prove eigenfunction expansions. Let $\Omega$ be the set of $\nu \in R$ for which $t(x, \mathfrak{l})$ is not defined. If we define $\hat{f}(\nu)$ as above for $\nu \in R \backslash \Omega$, then as in 6 .iii we will get a representation for $E(R \backslash \Omega)$, and we can handle $E(\Omega)$ separately. The other possibility is to represent $E(R \backslash \Lambda)$ by writing $R \backslash \Lambda$ as the union of sets $\Delta$ as above with pairwise disjoint interiors, and representing the projections $E(\Delta)$ by using different bases $t(x, \mathfrak{l})$ and different measures $d \rho(\nu)$ for each interval $\Delta$. We elaborate on the former approach.

TheOREM 6.iii. Let $H=\mathscr{L}+\mathscr{K}$ as above. Then:

(1) There is a function $t(x, \nu)=\left(t_{1}(x, \nu), \cdots, t_{n}(x, \nu)\right)$ such that

(a) $t(x, \nu)$ is defined for $\nu \in R \backslash \Omega$ where $\Omega$ is a closed countable set, and where defined

$$
L t(x, \nu)+\int_{\mathcal{F}} K(x, y) t(y, \nu) d y=\nu t(x, \nu),
$$

(b) for all $x \in \mathscr{I}$ and all $\nu \in R \backslash \Omega, t(x, \nu)$ is analytic in a neighborhood of $\nu$,

(2) there is a matrix-valued function $\rho(\nu), \nu \in R$, which is nondecreasing and is of bounded variation on compact subsets of $R$, 
(3) if $\hat{f}(\nu)=\int_{\mathscr{f}} t^{*}(x, \nu) f(x) d x$, and if $f \in R(E(R \backslash \Omega))$, then

$$
f(x)=\int_{R \backslash \Omega} t(x, \nu) d \rho(\nu) \hat{f}(\boldsymbol{\nu}),
$$

(4) the mapping $f \rightarrow \hat{f}$ is an isometry from $R(E(R \backslash \Omega)$ ) into $L^{2}(d \rho)$

(5) if $f \in D(H) \cap \mathscr{R}(E(R \backslash \Omega))$, then $\widehat{H f}(\nu)=\nu \hat{f}(\nu)$,

(6) if $\omega \in \Omega$, then dimension $\mathscr{R}(E(\omega))<\infty$, and if $\omega \in(\Omega \backslash \Lambda)$ then dimension. $\mathscr{R}(E(\omega)) \leqq n$.

Proof. We have already established (1.a) in Theorem 3.iv, while (b) follows easily from 5.i and 5.ii. Part (2) is simply a restatement of $5 . \mathrm{v}$, and (3) is immediate from 6.ii, as is (4). Theorem 6.ii also gives us the second part of (6), that is dimension $R(E(\omega)) \leqq n$ if $\omega \in(\Omega \backslash \Lambda)$. The other half of (6) follows quickly once we observe that every eigenfunction for $H$ must satisfy the differential equation $L \phi=\lambda \dot{\phi}$ on $\mathscr{J} \backslash B$.

To prove (5), note that $\chi_{\Delta} \widehat{H f}=\left[E_{H}(\Delta) H f\right]^{\wedge}$ for all compact intervals $\Delta$ in $R / \Omega$. Now for any compact interval $\Delta$ the operator $E_{H}(\Delta) H$ is bounded. Find a sequence $f_{n} \in C_{0}^{\infty}(\mathscr{F})$ such that $f_{n} \rightarrow f$. Since each $f_{n} \in D\left(S_{J}\right)$ for some $J$ we have $\widehat{H f}_{n}(\nu)=\nu \widehat{f}_{n}(\nu)$. Thus

$$
\begin{aligned}
\chi H f & =\left[E_{H}(\Delta) H f\right]^{\wedge}=\lim _{n \rightarrow \infty}\left[E_{H}(\Delta) H f_{n}\right]^{\wedge} \\
& =\lim _{n \rightarrow \infty} \chi_{L}(\nu) \nu \hat{f}_{n}(\nu)=\chi \nu \hat{f}(\nu) .
\end{aligned}
$$

Since $\Delta$ was an arbitrary compact interval in $R \backslash \Omega$, we are done.

THEOREM 6.iv. If $\Delta$ is as above, then the mapping $f \rightarrow \hat{f}$ from $R\left(E_{H}(\Delta)\right) \rightarrow L^{2}(\Delta, d \rho)$ is onto.

Proof. We modify part of the proof of Coddington and Dijksma [5], Theorem 6.5. Since the mapping is isometric we only need show the range is dense. Suppose that $\zeta \in L^{2}(\Delta, d \rho)$ is orthogonal to the

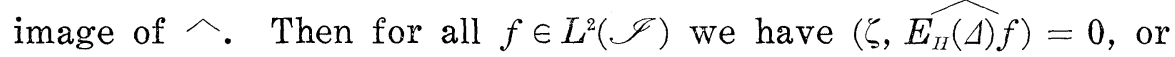

$$
0=\left(\zeta, \chi_{A} \hat{f}\right)=\int_{\Lambda}(t(x, \nu), f(x)) d \rho(\nu) \zeta(\nu)=0
$$

This means that if $\delta$ is a subinterval of $\Delta$, then

$$
0=\int_{\delta}(t(x, \nu), f(x)) d \rho(\nu) \zeta(\nu)
$$

Let $h_{i, n}(x)$ be a sequence of smooth nonnegative functions with integral one and support in $\left[c_{\imath}-1 / n, c_{\imath}+1 / n\right], n=1,2,3, \cdots$. Then we have 


$$
0=\int_{\delta}\left(t(\nu), h_{i, n}\right) d \rho(\nu) \zeta(\nu)
$$

Let $\delta_{i j}=1$ if $i=j$, and zero otherwise. Since $\left(t(\nu), h_{i, n}\right) \rightarrow$ $\left(\delta_{1 i}, \delta_{2 i}, \cdots, \delta_{n i}\right)$ uniformly in $\Delta$, we conclude that $0=\int_{\delta} d \rho(\nu) \zeta(\nu)$ for all intervals $\delta \subset \Delta$, which implies that $\zeta=0$.

THeOREM 6.v. Let $L=-D^{2}+q(x)$, where $q \in L_{1 \mathrm{oc}}^{\infty}(\mathscr{J})$ is real, and let $S_{J}$ and $J$ be as before. If $\mathscr{K}$ is compact, then $S_{J}$ has finitely many eigenvalues.

Proof. Let $\mathscr{L}$ be a self-adjoint realization of $L$ on $L^{2}(J)$. Suppose that $\lambda_{k} \in \sigma\left(S_{J}\right) \cap \rho(\mathscr{L})$. If $\phi_{k}$ is a corresponding eigenfunction of norm one we have

$$
(\mathscr{L}+\mathscr{K}) \phi_{k}=\lambda_{k} \phi_{k} \text {, or } \phi_{k}=-(\mathscr{L}-\lambda)^{-1} \mathscr{K} \phi_{k} .
$$

Since $\mathscr{L}$ is self-adjoint,

$$
\left\|\phi_{k}\right\| \leqq\left\|\left(\mathscr{L}-\lambda_{k} I\right)^{-1}\right\|\left\|K \phi_{k}\right\|=\frac{1}{\operatorname{dist}\left(\lambda_{k}, \sigma(\mathscr{L})\right)}\left\|\mathscr{K} \phi_{k}\right\| .
$$

Since $\mathscr{K}$ is compact we can conclude that as $k \rightarrow \infty$ the distance between the eigenvalues of $S_{J}$ and the spectrum of $\mathscr{L}$ must approach zero.

Since this statement is independent of $\mathscr{L}$, our conclusion is valid if we can find two self-adjoint realizations of $L$ whose spectra are uniformly separated off a compact set. For the cited Sturm-Liouville operators this is possible. See for instance Levitan and Gaszmov [10], page 23, where asymptotic estimates for the eigenvalues of these operators are given in terms of $q(x)$ and the boundary conditions.

VII. An example. Our restriction that $K(x, y)$ have compact support in $\mathscr{I} \times \mathscr{I}$ is a severe one, so we address ourselves to the question of what happens when this condition is relaxed. One of the results of Theorem 6 .iii was that every eigenvalue of $\mathscr{L}+\mathscr{K}$ had finite multiplicity. We will construct a bounded perturbation of a differential operator which is self-adjoint and which has a dense set of eigenvalues on the interval $(-\infty,-1)$, each of infinite multiplicity.

First some preliminaries. Recall that the essential spectrum of an operator $\mathscr{L}$ is $\{\lambda \in C \mid R(\mathscr{L}-\lambda I)$ is not closed $\}$. If $L$ is a formal differential operator, we define the essential spectrum of $L$ to be the essential spectrum of the minimal operator for $L$. From Dunford and Schwartz [7], page 1436, we have the following. 
$\lambda$ is in the essential spectrum of $L$ if and only if there is a bounded sequence $\left\{f_{n}\right\}$ of functions in the domain of the minimal operator $T$ corresponding to $L$ and $L^{2}(\mathscr{F})$ such that $\left\{(L-\lambda) f_{n}\right\}$ converges in $L^{2}(\mathscr{I})$, but $\left(f_{n}\right)$ has no strongly convergent subsequence.

LEMMA 7.ii. Given T, $L$ and $\left\{f_{n}\right\}$ as in Lemma 7.i and $\lambda$ in the essential spectrum of $L$, there is a sequence $\left\{g_{n}\right\}$ in $D(T)$ such that $\left\|g_{n}\right\|=1$ and $\left\|(L-\lambda) g_{n}\right\| \rightarrow 0$.

Proof. Since $\left\{f_{n}\right\}$ is not Cauchy there is some $\delta>0$ such that for all positive integers $N$ there are $k(N), m(N)$ such that $k(N)$, $m(N)>N$ and $\left\|f_{k(N)}-f_{m(N)}\right\|>\delta$. Define $h_{N}=f_{k(N)}-f_{m(N)}$. We then have $\delta<\left\|h_{N}\right\|$ and $\left\{h_{N}\right\}$ is bounded. Simply define $g_{N}=h_{N} /\left\|h_{N}\right\|$.

To construct our example we will choose $L=D^{2}$ on $L^{2}(R)$. It is known that the essential spectrum of $L$ is $\{\lambda \in R \mid \lambda \leqq 0\}$. Since the minimal operator is the closure of $L$ restricted to $C_{0}^{\infty}(R)$ we can find, for $\lambda<-1$,

(*) $\quad f_{\lambda} \in C_{0}^{\infty}(R)$ such that $\left\|f_{\lambda}\right\|=1$ and $\left\|(L-\lambda) f_{\lambda}-f_{\lambda}\right\|^{2}<\frac{1}{4}$.

Now observe that if $f_{\lambda}(x)$ satisfies $\left(^{*}\right)$, so does $f_{\lambda}(x+t)$ for all $t \in R$.

We proceed as follows. For $k$ a prime integer define $k Z=\{k z \mid z$ a positive integer\}. Choose an enumeration of the rationals less than -1 , denoted by $\left\{q_{n}, n=1,2, \cdots\right\}$. Find bijections $b_{k}$ from $D\left(b_{k}\right)=$ $k Z \backslash\left\{\bigcup_{n} n Z \mid n\right.$ is prime and less than $\left.k\right\}$ to $\left\{q_{n}\right\}$. Now define a mapping $b$ from the integers greater than or equal to 2 to $\left\{q_{n}\right\}$ by $b(m)=$ $b_{k}(m)$ if $m \in D\left(b_{k}\right)$. We then have a function from the integers to the rationals such that every rational less than -1 is the image of infinitely many integers. Define $\lambda_{i}=b(i)$.

Now find $f_{\lambda_{1}}$ satisfying (*). Define $K_{1}: L^{2}(R) \rightarrow L^{2}(R)$ by $K_{1} g=$ $-\left(L-\lambda_{1}\right) f_{\lambda_{1}}\left(g,\left(L-\lambda_{1}\right) f_{\lambda_{1}}\right) /\left(f_{\lambda_{1}},\left(L-\lambda_{1}\right) f_{\lambda_{1}}\right)$. Since $\left\|\left(L-\lambda_{1}\right) f_{\lambda_{1}}-f_{\lambda_{1}}\right\|^{2}<1 / 4$ we have

$$
\left\|\left(\left(L-\lambda_{1}\right) f_{\lambda_{1}}, f_{\lambda_{1}}\right) f_{\lambda_{1}}-f_{\lambda_{1}}\right\|^{2}<\frac{1}{4}
$$

or

$$
\left|\left(\left(L-\lambda_{1}\right) f_{\lambda_{1}}, f_{\lambda_{1}}\right)-1\right|^{2}<\frac{1}{4},
$$

so that

$$
\left|\left(\left(L-\lambda_{1}\right) f_{\lambda_{1}}, f_{\lambda_{1}}\right)\right|>\frac{1}{2}
$$


Thus $\left\|K_{1}\right\| \leqq 2\left\|\left(L-\lambda_{1}\right) f_{\lambda_{1}}\right\|^{2} \leqq 2 \cdot(9 / 4) \leqq 5$ by the triangle inequality. Now inductively define $K_{n}$ exactly as $K_{1}$ was defined, replacing $\lambda_{1}$ by $\lambda_{n}, f_{\lambda_{1}}$ by $f_{\lambda_{n}}$, and insisting that the functions $f_{\lambda_{1}}, i \leqq n$, have pairwise disjoint supports. We can insist on this last condition because of the remark immediately following $(*)$. Define an operator $K$ by $K f=\sum_{n=1}^{\infty} K_{n} f$. If we choose an orthonormal basis for $L^{2}(R)$ which includes $\left\{\left(L-\lambda_{i}\right) f_{\lambda_{i}}\right\}$, then the matrix representation for $K$ is diagonal, and each diagonal entry had absolute value bounded by 5 . If the functions $f_{\lambda_{i}}$ are chosen real, then $K$ is obviously self-adjoint and $\|K\| \leqq 5$.

Now notice that $L+K$ can be realized as a self-adjoint operator of $L^{2}(R)$, but if $\mu \in\left\{q_{n}\right\}$, then $\mu$ is an eigenvalue of $L+K$ of infinite multiplicity.

\section{REFERENCES}

1. N. I. Akhiezer and I. M. Glazman, Theory of Linear Operators in Hilbert Space, Fredrick Ungar, New York, 1961.

2. E. A. Coddington, Generalized resolutions of the identity for symmetric ordinary differential operators, Ann. of Math., 68 (1958), 378-392.

3. - Extension theory of formally normal and symmetric subspaces, Mem. Amer. Math. Soc., 134 (1973).

4. - Self-adjoint problems for nondensely defined ordinary differential operators and their eigenfunction expansions, Advances in Math., 14 (1974).

5. E. A. Coddington and A. Dijksma, Self-adjoint subspaces and eigenfunction expansions for ordinary differential subspaces, J. Differential Equation, 20 (1976).

6. E. A. Coddington and N. Levinson, Theory of Ordinary Differential Equations, McGraw-Hill, New York, 1955.

7. N. Dunford and J. T. Schwartz, Linear Operators, Part II, Interscience, New York, 1963.

8. S. Goldberg, Unbounded Linear Operators, McGraw-Hill, New York, 1966.

9. T. Kato, Perturbation Theory for Linear Operators, McGraw-Hill, New York, 1966.

10. B. M. Levitan and M. G. Gaszmov, Determination of a differential equation by two of its spectra, Russian Math. Surveys, 19 (1964).

Received January 21, 1978.

The University OF UtaH

SALT LAKE City, UT 84112 



\title{
PACIFIC JOURNAL OF MATHEMATICS
}

\section{EDITORS}

\author{
DONALD BABBITT (Managing Editor) \\ University of California \\ Los Angeles, CA 90024 \\ HUGo ROSSI \\ University of Utah \\ Salt Lake City, UT 84112 \\ C. C. MOORE \\ University of California \\ Berkeley, CA 94720
}

J. DugundJI

Department of Mathematics

University of Southern California

Los Angeles, CA 90007

R. FinN and J. Milgram

Stanford University

Stanford, CA 94305

\section{ASSOCIATE EDITORS}
E. F. BECKENBACH
B. H. Neumann
F. WolF
K. YOSHIDA

\section{SUPPORTING INSTITUTIONS}

\author{
UNIVERSITY OF BRITISH COLUMBIA \\ CALIFORNIA INSTITUTE OF TECHNOLOGY \\ UNIVERSITY OF CALIFORNIA \\ MONTANA STATE UNIVERSITY \\ UNIVERSITY OF NEVADA, RENO \\ NEW MEXICO STATE UNIVERSITY \\ OREGON STATE UNIVERSITY \\ UNIVERSITY OF OREGON
}

UNIVERSITY OF SOUTHERN CALIFORNIA

STANFORD UNIVERSITY

UNIVERSITY OF HAWAII

UNIVERSITY OF TOKYO

UNIVERSITY OF UTAH

WASHINGTON STATE UNIVERSITY

UNIVERSITY OF WASHINGTON

The Supporting Institutions listed above contribute to the cost of publication of this Journal, but they are not owners or publishers and have no responsibility for its content or policies.

Mathematical papers intended for publication in the Pacific Journal of Mathematics should be in typed form or offset-reproduced, (not dittoed), double spaced with large margins. Please do not use built up fractions in the text of the manuscript. However, you may use them in the displayed equations. Underline Greek letters in red, German in green, and script in blue. The first paragraph or two must be capable of being used separately as a synopsis of the entire paper. Please propose a heading for the odd numbered pages of less than 35 characters. Manuscripts, in triplicate, may be sent to any one of the editors. Please classify according to the scheme of Math. Reviews, Index to Vol. 39. Supply name and address of author to whom proofs should be sent. All other communications should be addressed to the managing editor, or Elaine Barth, University of California, Los Angeles, California, 90024.

50 reprints to each author are provided free for each article, only if page charges have been substantially paid. Additional copies may be obtained at cost in multiples of 50 .

The Pacific Journal of Mathematics is issued monthly as of January 1966. Regular subscription rate: $\$ 72.00$ a year (6 Vols., 12 issues). Special rate: $\$ 36.00$ a year to individual members of supporting institutions.

Subscriptions, orders for numbers issued in the last three calendar years, and changes of address should be sent to Pacific Journal of Mathematics, P.O. Box 969, Carmel Valley, CA 93924, U.S.A. Older back numbers obtainable from Kraus Periodicals Co., Route 100, Millwood, NY 10546.

PUBLISHED BY PACIFIC JOURNAL OF MATHEMATICS, A NON-PROFIT CORPORATION

Printed at Kokusai Bunken Insatsusha (International Academic Printing Co., Ltd.). 8-8, 3-chome, Takadanobaba, Shinjuku-ku, Tokyo 160, Japan.

Copyright (C) 1979 by Pacific Journal of Mathematics Manufactured and first issued in Japan 


\section{Pacific Journal of Mathematics}

\section{Vol. 81, No. $2 \quad$ December, 1979}

Ersan Akyildiz, Vector fields and equivariant bundles ............... 283

Ehrhard Behrends, The centralizer of tensor products of Banach spaces ( $a$ function space representation) ......................... 291

Geoffrey R. Burton, Congruent sections of a convex body ............. 303

John Warnock Carlson, $H$-closed and countably compact extensions ...... 317

Robert Charles Carlson, Eigenfunction expansions for selfadjoint

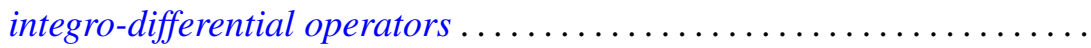

Robert Damiano, Coflat rings and modules.

Eric Karel van Douwen and Washek (Vaclav) Frantisek Pfeffer, Some properties of the Sorgenfrey line and related spaces ................

Uri Elias, Necessary conditions and sufficient conditions for disfocality and

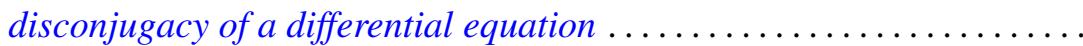

V. L. (Vagn Lundsgaard) Hansen, Polynomial covering spaces and homomorphisms into the braid groups .......................

Paul Hess, Dedekind's problem: monotone Boolean functions on the lattice

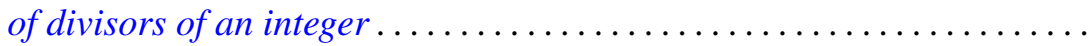

Alan Hopenwasser and David Royal Larson, The carrier space of a reflexive

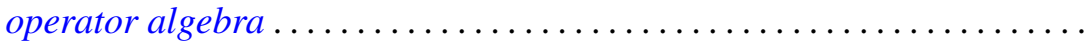

Kyung Bai Lee, Spaces in which compacta are uniformly regular $G_{\delta} \ldots \ldots$.

Claude Levesque, A class of fundamental units and some classes of

Jacobi-Perron algorithms in pure cubic fields...

Teck Cheong Lim, A constructive proof of the infinite version of the

Belluce-Kirk theorem ..........................

Dorothy Maharam and A. H. Stone, Borel boxes

Roger McCann, Asymptotically stable dynamical systems are linear

Peter A. McCoy, Approximation and harmonic continuation of axially symmetric potentials in $E^{3} \ldots \ldots \ldots \ldots \ldots \ldots \ldots$

Takahiko Nakazi, Extended weak-* Dirichlet algebras ....

Carl L. Prather, On the zeros of derivatives of balanced trigonometric polynomials ................................

Iain Raeburn, An implicit function theorem in Banach spaces...

Louis Jackson Ratliff, Jr., Two theorems on the prime divisors of zeros in completions of local domains...

Gloria Jean Tashjian, Cartesian-closed coreflective subcategories of

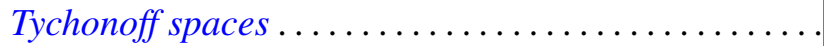

Stephen Edwin Wilson, Operators over regular maps.... . . 